\title{
A01-25225
}

42nd AIAAIASMEJASCEJAHSIASC Structures, Structural Dynamics, and Materials Conference and Seattle, WA Exhibit 16-19 April 2001
AIAA-2001-1485

\section{Damage Tolerance of Cracked Cylindrical Shells under Internal Pressure}

\author{
Dennis Hoogkamer, Anthony M. Waas* and Johann Arbocz ${ }^{\dagger}$ \\ Composite Structures Laboratory, \\ Department of Aerospace Engineering, \\ University of Michigan, Ann Arbor, MI48109-2140 \\ February 9, 2001
}

\begin{abstract}
In the present work, a series of unstiffened, thinwalled (radius to thickness ratio, $R / t=216$ ), metallic cylindrical shells, containing centralized cracks at angles to the shell generators ranging from $0^{\circ}$ to $90^{\circ}$ at intervals of $15^{\circ}$ and of two crack lengths, are internally pressurized in order to determine the effect of crack angle on the failure mechanism of the shells. This is done under two different sets of boundary conditions. In the first case the shell is free to move in the axial direction, in the second case this axial movement is constrained.

Curves of the maximum pressure leading to global failure of the shells as a function of crack angle for two different crack lengths and for both cases of boundaray consitions are presented. The present experimental data can be used to find a governing predictive crack growth criterion as a function of crack angle. This criterion can be used in commercial finite element codes for predicting the failure of more complex shell structures like those used in transport fuselage shell structures.

The experimental data are explained via a fracture mechanics based analysis. For cracks which are inclined with respect to the applied stress, both the opening (tensile) mode I stress intensity factor and the sliding (shear) mode II stress intensity factor are significant. These depend on the angle of the crack with respect to the applied load. Fairly good agreement between analysis and experiment is obtained if adjusted values of $K_{I_{c}}$ and $K_{I I_{c}}$ are used along with the assumption that crack growth is governed by a quadratic failure criterion. The adjusted values account for shell curva-
\end{abstract}

\footnotetext{
${ }^{*}$ Graduate student and Professor of Aerospace Engineering respectively, University of Michigan. Assoc. Fellow AIAA, Copyright (C)2001 by Anthony M. Waas. Published by the AIAA, with permission.

${ }^{\dagger}$ Chairman, Aerospace Structures \& Computational Mechanics Group, Department of Aerospace Engineering, Delft University of Technology, The Netherlands. Fellow AIAA.
}

ture through a bulging factor $\beta$. Finite element simulations of selected shell configurations are carried out to obtain the crack flange deflection corresponding to the burst pressures. An approximate analysis of $0^{\circ}$ angle cracks based on technical beam theory and axisymmetric cylindrical shell analysis was presented earlier [1]. Results from this analysis are compared with the present finite element analysis for $0^{\circ}$ angle cracks in a forthcoming paper [2]. Finally, images of the crack propagation event acquired at high framing rates for selected shell configurations are included.

\section{Introduction}

Due to the aging of the commercial aircraft fleet of the world's airlines, it is becoming more likely that aircraft do not meet their original intended design requirements due to the onset of fatigue cracks and damage caused by either corrosion or impact. The effects of this multiple site damage can be devastating as was shown in Aloha flight 243 where a considerable part of the fuselage roof was blown away due to multiple site fatigue damage [3]. The concept of damage tolerance is based on the notion of assessing the departure in response of a structure that contains a specified amount of damage (imperfections) in comparison with the intended response of the corresponding undamaged structure. Thus, in a sense, damage tolerance provides an assessment of how 'robust' a structural design or concept is. The 'amount' and 'type' of damage is specified a priori, based on the knowledge of the life-cycle that is specific to the particular aircraft or spacecraft.

At The University of Michigan, Department of Aerospace Engineering, Composite Structures laboratory, a program has been initiated with the aim of investigating the static and dynamic failure of cylindrical shells containing cracks of different lengths and at dif- 
ferent angles to the shell generators. In the present paper, experimental results are presented to characterize the failure of a set of cylindrical metallic shells containing a centralized crack at an angle with respect to the generators of the shell. Results from a finite element analysis for $0^{0}$ angle cracks are also presented.

Previous work done by a handful of authors has mainly been focussed towards the static loading (uniand biaxial) of a cylindrical shell with a centralized crack at a zero angle with respect to the shell generators. These previous studies can roughly be split up into an experimental and a numerical group, however the most significant difference between the different research initiatives is the use of a linear or a nonlinear approach in analyzing the results, $[4,5,6]$.

Linear fracture mechanics suggest that the likelihood of fracture depends on the crack tip stress intensity factor reaching a critical value. It is assumed initially that changes in the shell geometry during the event of loading do not cause any changes in the crack tip stress intensity factor. After solving the problem using these assumptions, a so-called bulging factor is implemented to account for nonlinear effects which occur due to local shell bending deformation. The bulging factor provides a characterization of the departure in the stress intensity factor due to shell wall curvature. Thus,

$$
\beta=\frac{k_{s h e l l}}{k_{p}},
$$

where $k_{p}$ is the stress intensity factor for the corresponding flat plate crack problem.

Analytical methods that have been reported previously are generally only valid for small $(\leq 5)$ shell curvature parameters $\lambda_{i s o}$, where

$$
\lambda_{i s o}=\frac{a}{\sqrt{R t}} \sqrt[4]{12\left(1-\nu^{2}\right)} .
$$

In the above expression, $2 a$ is the crack length, $R$, the shell radius, $t$ is the shell wall thickness and $\nu$ the poisson's ratio of the isotropic shell wall material. These methods tend to overestimate the physical bulging unless the investigated cracks are small. The cause of the over estimation is the linearization of shell equilibrium equations. The linearization does not account for the tensile stresses which develop parallel to the crack line and increase the resistance to bulging and crack opening [7].

All nonlinear work that has been done in the field of cylindrical shells with centralized cracks are focussed towards specific materials and are valid under special conditions. Previous work by Starnes and Rose [7] summarizes the resulting semi-empirical relations of these research initiatives and refutes or verifies them using a nonlinear Finite Element code-STAGS (Structural Analysis of General Shells).

Starnes and Rose [7] have shown that for a shell with a small $\lambda_{\text {iso }} \leq 5$, the bulging radial displacement $w_{c}$, measured at the crack flange center is a linear function of the far-field hoop stress. They also found that the bulging factor was independent of the hoop stress (pressure) for shells with a small $\lambda_{i s o}$ and the nonlinear stress intensity factor of the shell is a linear function of the far-field hoop stress. For shells with a large shell curvature parameter $(\geq 10)$, the results obtained were significantly different. The radial bulging displacement now is a nonlinear function of the circumferential stress. Further, as the load is increased, axial stresses along the crack edge become more dominant resulting in a membrane tensile axial stress, which increases the resistance to bulging even more.

The results presented by Starnes, Rose and Young [8] includes finite element results of the effect of combined loading on the nonlinear bulging effect in shells of different configurations, with cracks in both axial and circumferential directions.

The present paper is organized as follows. In section 2 , details of the experimental program carried out to obtain a failure envelope for cracked cylindrical shells are presented. Included in this section is also a description of the work that was done to obtain insight into the dynamics of the failure process. Crack path direction and crack velocity have been measured for selected shell configurations.

In section 3 , details of the finite element simulation that was carried out to understand the cracked shell response are presented. Results are presented for crack orientations at $\theta=0^{\circ}$, where $\theta$ is measured from the shell generators.

For this case, the predictions of an analytical model for crack face bulging developed earlier [1], using technical beam theory and an assumption of axisymmetry are compared against the results presented by Starnes and Rose[7] and the present finite element work in [2]. A Fracture Mechanics based failure criterion is introduced in section 4. Finally, some concluding remarks are presented in section 5 .

\section{Experimental Work}

For all of the experimental work reported in this paper, seamless shells, previously used as containers for Coca-Cola beverages were used. Due to the high manufacturing tolerances of the containers, the shells are easily machined into a cylindrical shell by removing the two ends of the containers. As a result of the production process that is used to produce beverage contain- 
ers, a certain thickness distribution over the length of the shell is apparent. The thickness distribution is displayed in Figure 2.1. The 'nominal' wall thickness is 0.006 inches and the 'nominal' internal radius is 1.3 inches. This thickness distribution does not seem to be of high significance due to the fact that most of the thickness variation falls inside the clamps holding the shell. In addition, the presence of a relatively large crack overwhelms the presence of other unintended imperfections such as a non-uniform thickness distribution. As shown in the results section of this paper, consistent burst pressures for several of the shells are repeatedly obtained.

The shells are placed between a static base and a mobile top plate, as shown in Figure 2.2. Two types of tests were conducted. In the first, the top plate was free to move when the shells were pressurized. In the second, the top plate was held from moving outwards during the pressurization. The shell is clamped to the base and top plate by accurately manufactured, adjustable clamps leaving a free length of the shells in between the clamps of 3.5 inches. Nitrogen gas is used to pressurize the shell and kept from flowing out by two o-rings, placed between the shells and the clamps at both ends as shown schematically in Figure 2.2. The adjustable rings are kept from damaging the outside of the shell by wrapping a piece of Teflon tape at the outside end of the shell. Note that in some of the tests, where the top plug is free to move, results obtained correspond to a situation of pure internal pressure. This situation is obtained by allowing the top clamp to axially move outward during the pressurization process. However it was found that the entire shell (and the top clamp) "slid out" from the bottom clamp during some of the tests, thereby relieving the axial stress in the shell. This observation was captured by the high speed images that were taken during the pressurization process. This characteristic of the setup results in an axial load that is close to being equal to zero. The pure internal pressure loading reduces the complexity of the problem of the far field stress resultants which are now entirely a circumferential hoop stress component. Closer to the crack, bending of the crack flanges introduces other stress and moment resultants that produce a departure from the pure hoop stress case. In the second set of tests, the axial motion of the top plug is constrained from moving outward by a plate, which is attached to a loadcell in order to be able to measure the axial load. This second set of boundary conditions is more specific to achieving near zero axial elongation during the pressurization process. That is, the two ends of the shell are restrained from moving axially during the pressurization, by a clamping ring.

The base is connected to a pressure transducer and an amplifier, which are connected to a high speed oscilloscope. As a result, the pressure is measured with an accuracy of 0.3 psi. Finally, the crack in the shell is sealed from inside with a very thin sheet of plastic, to prevent the gas leaking through the crack. The plastic sheet is glued at its corners to the inside of the shell to ensure that it remains in place, but at the same time will not influence the properties of the shell locally at the crack tip. When conducting a test, the Nitrogen gas is released from a gas cylinder into a reservoir at high pressure after which a high speed electric valve releases the pressure into the shell. This approach makes it possible to 'instantly' pressurize the shell. A typical pressure versus time history plot for sudden pressurization is as indicated in Figure 2.3. A strain gage placed at one of the crack tips is used to trigger an ultra high speed variable framing rate digital camera(CORDIN), which is used to capture images of the crack propagation process (Figure 2.4 and Figure 2.5). Some of these results will be discussed later. One of the objectives of the experimental work is to determine the dynamic crack growth characteristics when the shells are exploded by "overpressurization". For these tests, the straingage used to monitor the the strain at the crack tip is also used to trigger the CORDIN camera ( 8 frames at $0.5 \mu$ sec. between frames, with a $0.01 \mu$ sec. exposure time) that can acquire images which will show the events in the vicinity of the crack tip at the onset of tearing.

\subsection{Proposed Methodology}

The main objective of the experiments was to obtain an experimental correlation between the cracking pressure, the crack angle and the crack length. This was done by considering different crack lengths, each for various crack angles, as defined in Figure 2.6.

In [7], a non-dimensional crack curvature parameter is defined for isotropic shells as $\lambda=a / \sqrt{R t}$, where $2 a$ is the crack length, and $R$ and $t$ are the radius and thickness of the shell respectively. We will use this definition of $\lambda=a / \sqrt{R t}$, throughout this paper. The dimensions of the shells as given in [7] are compared to the shells that were used in the present experiments in Table 2.1. The equivalent crack lengths for the present experiments are then calculated such that the non-dimensional crack curvature parameters, $\lambda$, for the shells with $\theta=0$, are similar to those corresponding to the shells used in [7]. The equivalent crack lengths are presented in Table 2.2. The crack angles that were machined are displayed in Table 2.1. The method of producing the cracks has a large impact on the "quality" of the crack tip. In the present case the cracks are all produced using a rigid fixture in which the shell is 
placed aver a 'snuggly' fitting mandrel and an exact-o knife is implemented to produce the crack. A magnified photomicrograph of the crack tip is presented in Figure 2.7. A typical crack tip radius is indicated as $(0.0011$ inches). This radius is $16 \%$ of the shell wall thickness, and $0.16 \%$ and $0.12 \%$ of the initial crack lengths of 0.668 and 0.886 inches respectively. This is representative of the "sharpness" of the cracks that are studied in the present experiments. Note that the definition of the non-dimensional crack curvature does not include the free length of the shell, and hence the ratios for $r / L$ and $a / L$ for the shells used in the present experiments are not compared to the corresponding ratios as used in [7]. It is expected that the effect of these parameters on the cracking pressures is of a lot lower significance than the other parameters, since $\frac{a}{L} \ll 1$.

When studying the preliminary images that were obtained using the CORDIN camera, it becomes apparent that the crack opens significantly before it starts growing in an unstable manner. This indicates that a nonlinear phenomenon with respect to the loading, possibly due to crack tip plasticity is predominant and thus this will have to be implemented in modelling. The peak pressures that are reported in the present paper(point A in Figure 2.3) were read off as is indicated in Figure 2.3. However, it is not certain that at this pressure the crack starts growing in a stable or unstable manner or that perhaps the crack starts to grow at a lower pressure (like point B, as indicated in Figure 2.3). In order to clarify these uncertainties a more detailed study is presently underway using high speed photography and using more refined ways of synchronizing the pressure transducer output and the image-time history data.

\subsection{Experimental Results}

Figure 2.8 shows the results for the peak pressures that were obtained for identical crack lengths (equivalent to the 3 and 4 inch crack lengths as presented in [7]), but for several crack angles. Note that unlike what one would expect for a crack in a "flat sheet", the pressure at which the shell catastrophically fails is about 6 times higher when the crack is in the hoop direction (90 deg.) than when the crack is in the axial direction ( 0 deg.). A linear fracture mechanics based analysis of the experimental data indicates that a quadratic crack growth criterion is able to capture the qualitative trends that are indicated by the experimental results. This analysis is presented later.

In Figure 2.9, the crack tip velocity is set out against time, where the time origin is chosen at the peak where the crack tip strain starts to relax, for a shell containing a crack at $\theta=0$ and loaded by a sudden increase of internal pressure. These measurements were made with the images obtained via the CORDIN camera. The first frame in Figure 2.4 corresponds to a crack length of 1.1 inches at $t=0$. Note that the initial unpressurized crack length is 0.6 inch. The pictures are snap shots in a window of time of the crack propagation event. The crack tip velocity versus time in this window provide insight into the non-uniform crack propagation event. The crack tip velocity increases with a exponential type behavior. This shows that the crack accelerates in a nonuniform manner while it is propagating. If the shells were long enough, it would be interesting to determine the terminal (asymptotic) velocity that the crack will finally reach. Dynamic crack growth in flat metallic structures have been a hot bed of research for sometime now and the interested reader is referred to [10], for more details pertaining to dynamic fracture mechanics.

\section{$3 \quad$ Finite Element Analysis}

A Finite Element Analysis to simulate the pressurized shell experiments was conducted using the commercial FEA package ABAQUS with Hypermesh as the preprocessor. Other packages were investigated for preprocessing, but none were as effective as Hypermesh. A mesh of the entire shell was made using S8R, 8node quadrilateral doubly curved shell elements, implemented using reduced integration. These elements allow for through the thickness shear deformation, which can be significant for high deformation zones. Further, mesh refinement was achieved using 6-node, STRI65, triangular shell elements far away from the high deformation regions. The STRI65 are 6-node triangular shell elements, using 5 degrees of freedom per node and implemented using reduced integration. This type of mesh refinement was chosen over the use of a multiple point constraint approach due to the recommendations provided in the ABAQUS manual [12]. The principle reason is that MPC's introduce constraints that lock the response in the finer mesh.

Due to the difficulties associated with meshing a curved surface, the shell was constructed by modelling a flat sheet with the imperfection present (the crack) and then have ABAQUS convert the given coordinates to a cylindrical coordinate system. This resulted in inserting $\mathrm{x}, \mathrm{y}, \mathrm{z}$-coordinates where the $\mathrm{x}$ coordinate was the radius of the shell, the y coordinate was the circumferential distance and the $\mathrm{z}$ coordinate, the coordinate along the axial direction of the shell.

\subsection{Mesh Sensitivity}

As part of the finite element work, a mesh sensitivity study was conducted. A shell with a longitudinal 
crack was modelled using three different mesh densities. Mainly, the area around the imperfection was modelled with a finer/coarser mesh, but also the density of elements in the far field was varied in these three cases. Table 3.1 indicates the results of this study. All further finite element analyses were conducted with a mesh density as is indicated in table 3.1. Note that all the models used in the mesh sensitivity study involved geometric nonlinearity as well as material nonlinearity (J2 incremental theory of plasticity) to create a worst case scenario. As indicated in Table 3.1, the normalized crack flange deflection $\left(\frac{W_{c}}{t}\right)$, has recahed a converged value with the second mesh containing 167,748 degrees of freedom. Thus, further studies were carried out with this less dense mesh.

\subsection{Modelling Approach}

The finite element analysis was conducted using the NLGEOM function in ABAQUS which incorporates geometric nonlinearity. This is needed because of the relatively large rotations near the crak flanges of the shell. The pressure loading is gradually built up in a fashion similar to the pressure buildup during the tests, allowing ABAQUS to reduce the step size for it to reach convergence. For purposes of comparison, a geometrically and materially linear analysis was also carried out. Note that symmetry with respect to crack position, in the case of the $0^{\circ}$ degree cracks was not implemented since future work will contemplate the use of cohesive zone modeling [11], to provide crack propagation trajectories. In such an approach, the crack growth direction is determined as a part of the solution and not asumed a priori.

\subsection{Material properties}

The material properties of the shell wall material were determined by doing several tensile tests on dogbone specimens cut out of the shell wall. These tests resulted in a stress strain curve which is represented in Figure 3.1. The test data were fitted with a Ramberg-Osgood formula:

$$
\epsilon=\left(\frac{\sigma}{E}\right)+\left(\frac{\sigma}{H}\right)^{\frac{1}{n}}
$$

The two unknowns in the above relation are found by obtaining the best fit to the test data. Note that the above representation is convenient since it splits the total starin into an elastic and plastic part. This decomposition is conveneient for the implementation of J2 inceremental flow theory of plasticity for an isotropic solid as provided in built-in subroutines within ABAQUS. The Young's modulus, Poisson's ratio and yield stress for the shell material were found to be, $E=24.43 \mathrm{GPa}$, $\nu=0.30$, and $\sigma_{y i e l d}=51 \mathrm{MPa}$ ), respectively. Note that recycled aluminum has degraded properties compared to virgin aluminum.

\subsection{Results}

To compare the results for the different model characteristics and different crack configurations, the normalized crack face bulging displacement, $\frac{w_{c}}{t}$, is plotted as a function of normalized hoop stress in Figure 3.2. This figure indicates the results separated by crack configuration. As it can be observed for the cases of pure elastic material (without any plasticity included), all the crack configurations for which finite element analysis was performed, the relation between the central crack flange deflection and the hoop stress is approximately linear. The nonlinear geometry function in ABAQUS doesn't initiate a nonlinear response (yet) for the pressures at which the shells fail in the experiments. This is consistent for shells of 'small' $\lambda$ as was discussed by Starnes and Rose in [7]. The value for the present shell is $\lambda=5$. Figure 3.3 shows the deformed mesh of the $0^{\circ}$, crack case.

The picture is very different for models which include material nonlinearity. Here the nonlinearity effect (of the material this time) kicks in at the point where the plastic curve departs from the elastic curve. The image of rapidly increasing $\frac{w_{c}}{t}$ is a result of the decreasing stiffness of the crack flange material loaded into the plastic range as can be observed from the stress-strain curve in Figure 3.1. The stiffness of the material is represented by the slope of the curve, which clearly reduces significantly well into the plastic regime of the material. The stiffness reduces so much that at a specific loading, the rotational increments that are a result of the ramp loading (that is inherent in any geometrically nonlinear model)are too large for ABAQUS to handle, even when time increments that are automatically reduced by ABAQUS to values that would make a single analysis run for a long time. ABAQUS then judges convergence unlikely and discontinues the analysis.

Further, it is interesting to note that the effect of axially constraining the boundaries of the shell has a very small stiffening effect on the shell as can be observed in Figure 3.2 for the fully elastic material cases. However, this effect is larger when the material is modelled with plastic stress-strain characteristics included. The reason for doing analyses including the axial constraints are related to the test setup, which does not allow for the boundaries to move in the negative direction (outward, axially). However, the clamping ring in the experiment may allow the shell to contract during the pressurization of the shell. This aspect is not modelled here. 
To put all the numerical results in perspective, let us define a factor,

$$
\beta^{*}=\frac{w_{c_{\text {nonlinear }}}}{w_{c_{\text {linear }}}}
$$

This factor compares the crack flange deflection from a purely linear elastic (material and geometry) point of view, against a fully nonlinear (material and geometry) point of view, as a function of loading (pressure). $\beta^{*}$ against pressure is plotted in Figure 3.4, for all of the cases $\left(\theta=0^{\circ}, 45^{\circ}\right.$ and $\left.90^{\circ}\right)$. Also, we have marked by a 'dashed' line the conjectured trajectories that we would have expected as the pressure is further increased. Notice that $\beta^{*}$ begins to exponentially increase at about $\sigma_{\theta} / E \simeq 0.0025-0.0030$. This shows that for this range of pressure, $\beta^{*}$ becomes unbounded. Further, when we examine the stresses ahead of the crack tip as a function of distance from the crack tip (Figure 3.5), we notice that a very large region of the shell is substantially plastic even at $43 \%$ of the burst pressure for the $\theta=90^{\circ}$ case. Thus, the current static Finite Element results yield that the maximum hoop stress sustainable by the shells for the cracks studied are $\sigma_{\theta} / E \simeq 0.0030$. This number is higher than the hoop stress corresponsing to the experimental burst pressure for $\theta=0^{\circ}$ case, but lower than the experimental case for $\theta=90^{\circ}$.

Some of the differences in the experimental results and the finite element results are due to the noninclusion of dynamic effects associated with the experiments and due to the uncertainty in associating the maximum measured pressure as the burst pressure. As stated earlier, it is quite possible that crack propagation occurs while the pressure is still increasing, since the loading mechanism is "over pressurization". Further, it is known that metals are strain rate sensitive, with the material properties substantially "stiffer" when rate effects are incorporated. The incorporation of these three effects, namely, (a) certainty in establishing the exact pressure at first crack propagation, (b) inclusion of dynamic effects and (c) inclusion of rate dependent material properties are relegated to future work.

\section{Linear Elastic Fracture Me- chanics}

\subsection{Introduction}

For cracks which are inclined with respect to the applied stress, as is indicated in Figure 2.6, the opening(tensile) mode I stress intensity factor and the sliding(shear) mode II stress intensity factor are significant. These depend on the angle of the crack with respect to the applied load. Consider the configuration corresponding to the flat plate equivalent as shown in Figure 2.6, subjected to pure hoop stress. Then, by transforming the stresses to coordinates that are aligned and normal to the crack, one obtains the following expressions for the mode I and mode II stress intensity factors (SIF's);

$$
\begin{gathered}
K_{I}=\sigma \sqrt{\pi a} \cos ^{2} \theta, \\
K_{I I}=\sigma \sqrt{\pi a} \sin \theta \cos \theta .
\end{gathered}
$$

If crack growth can be described by a quadratic failure criterion, it can be hypothesized that,

$$
\left(\frac{K_{I}}{K_{I_{c}}}\right)^{2}+\left(\frac{K_{I I}}{K_{I I_{\mathrm{c}}}}\right)^{2}=1
$$

where, $K_{I_{c}}$ is the critical mode I stress intensity factor and is a known quantity for a given material. $K_{I I_{c}}$ is taken to be $K_{I_{\mathrm{c}}}$ multiplied by a factor $\alpha$, and is the critical mode II stress intensity factor. Thus, assume that,

$$
K_{I I_{c}}=\alpha K_{I_{c}} .
$$

Substituting these in the above crack growth criterion, we obtain,

$$
K_{I}^{2}+\frac{1}{\alpha^{2}} K_{I I}^{2}=K_{I_{c}}^{2}
$$

With the relations for $K_{I}$ and $K_{I I}$ given above, this yields,

$$
\sigma^{2}(\pi a) \cos ^{4} \theta+\frac{1}{\alpha^{2}} \sigma^{2}(\pi a) \sin ^{2} \theta \cos ^{2} \theta=K_{I_{c}}^{2},
$$

as the condition describing crack growth. Simplification of this relation results in,

$$
\sigma^{2}=\left(\frac{K_{I_{c}}{ }^{2}}{c}\right)
$$

where,

$$
c=\pi a \cos ^{2} \theta\left(\cos ^{2} \theta+\frac{1}{\alpha^{2}} \sin ^{2} \theta\right)
$$

As a special case one can assume $\alpha$ to be equal to 1 . This results in a relation for the constant $c$ :

$$
c_{\alpha=1}=\pi a \cos ^{2} \theta
$$

This yields for $\sigma$,

$$
\sigma=\frac{K_{I_{c}}^{2}}{\sqrt{\pi a} \cos \theta} .
$$

We can now apply the above development to a cylindrical shell containing a crack at some angle to the shell generators, when subjected to internal pressure. To do so, we shall assume the shell radius $R \gg t$, where $t$ is 
the shell wall thickness. Then, the above 'flat plate' development is applicable. For pure internal pressure,

$$
\sigma=\frac{p R}{t}
$$

where $p$ is the internal pressure. Thus, the critical pressure for crack growth for a "flat plate" is obtained as,

$$
P_{c}=\left(\frac{t}{R}\right) \frac{K_{I_{c}}^{2}}{\sqrt{\pi a} \cos \theta} .
$$

With the definition of $\beta$, therefore, for a shell

$$
P_{c}^{\text {shell }}=\left(\frac{t}{R}\right) \frac{K_{I_{c}}^{2}}{\sqrt{\pi a} \cos \theta} \cdot \frac{1}{\beta^{2}} .
$$

When the experimental results are compared against the predicted results, the crack tip bulging can be computed and is found to be $\beta=3.39$

\subsection{Results}

Results obtained using the above formula for critical pressure are compared against the experimental results in Figure 4.1. In this Figure, two curves computed using two different values of $K_{I_{c}}$ are indicated. The first curve uses $K_{I_{c}}=22 K s i \sqrt{m}$ while the second curve is computed using the value of $K_{I_{c}}$ that would match the experimental result corresponding to $\theta=0$, which is the case of an axial crack. From this case we obtain, $\beta=3.39$. Note that, the first curve predictions are much higher than the experimental result, whereas the prediction value obtained by matching the experimental data is much lower. This apparent lowering is associated with the crack face bulging due to localized bending deformations. What is encouraging is that the predicted trend for critical pressure by the analysis matches what was obtained experimentally. The analysis has several limitations as pointed out earlier, the most important of which are the restriction to a mathematically sharp crack and the restriction to a 'flat plate'. These effects must be properly accounted for and the need to include geometrically nonlinear effects due to crack face bulging as pointed out in [9], is also appreciated.

\section{Concluding Remarks}

In this paper, a set of experimental results for the burst pressure of internally pressurized shells has been presented. Consistent results for 3 sets of shells corresponding to $\lambda=3.8$ and $\lambda=5.0$ were presented as a function of crack angle $\theta$. The experimental results for burst pressure show that cracks with $\theta=0^{\circ}$ are the most critical. Further the experimental results have

\begin{tabular}{|l|l|l|l|}
\hline Symbol & From [7] & From [7] & $\begin{array}{l}\text { Aluminum } \\
\text { Shells }\end{array}$ \\
\hline $\mathrm{R}$ & $9.0 \mathrm{in.}$ & $10 \mathrm{in.}$ & $1.30 \mathrm{in.}$ \\
$t_{1}$ & $0.020 \mathrm{in.}$ & $0.1 \mathrm{in.}$ & $0.006 \mathrm{in.}$ \\
$t_{2}$ & $0.040 \mathrm{in.}$ & $\ldots$ & $\ldots$ \\
$\mathrm{L}$ & $36 \mathrm{in.}$ & $20 \mathrm{in.}$ & $3.5 \mathrm{in.}$ \\
$\theta$ & $0 \mathrm{deg}$. & $0 \mathrm{deg}$. & $0,15,30$ \\
& & & $45,60,75$, \\
& & & $90 \mathrm{deg}$. \\
$2 \mathrm{a}$ & $1,2,3,4 \mathrm{in.}$ & $4 \mathrm{in.}$ & 0.668, \\
& & & $0.886 \mathrm{in.}$ \\
\hline
\end{tabular}

Table 2.1: Dimensions of Shells.

\begin{tabular}{|l|l|}
\hline $\begin{array}{l}\text { Crack lengths } \\
\text { From }[7]\end{array}$ & $\begin{array}{l}\text { Crack lengths } \\
\text { current shells }\end{array}$ \\
\hline 1.0 in. & $\ldots$ \\
2.0 in. & $\ldots$ \\
3.0 in. & 0.668 in. \\
4.0 in. & 0.886 in. \\
\hline
\end{tabular}

Table 2.2: Crack lengths for equal crack curvature parameters, $\theta=0 \mathrm{deg}$. resulting in equal $\lambda$

been used to obtain $\beta=3.39$ for the present tested shells.

The Finite Element Analysis of selected shell configurations were presented. Both geometrical and material nonlinearity were included. It was observed that substantial plasticity around the crack tip region leads to large crack flange deflections. Linear elastic analyses were carried out for all the cases succesfully. A non dimensional factor $\beta^{*}=\frac{w_{c_{\text {nenlinear }}}}{w_{c_{\text {linear }}}}$ was introduced to summarize the results. The plot of $\beta^{*}$ versus normalized hoop stress indicates that when the hoop stress reaches a range of $\sigma_{\theta} / E \simeq 0.0025-0.0030, \beta^{*} \rightarrow \infty$. This indicates that, with the given input and given pressure controlled (as opposed to volume controlled) loading, this is the maximum range of $\left(\sigma_{\theta} / E\right)$ that is possible. The reasons for the discrepancy between the experimental results and the FEA analysis for the $\theta>0^{\circ}$ cases was discussed earlier and it is felt that the inclusion of these effects will substantially improve this discrepancy. 


\begin{tabular}{|l|l|l|l|l|}
\hline $\begin{array}{l}\text { Crack } \\
\text { Angle }\end{array}$ & element type & no. d.o.f. & $\begin{array}{l}\text { Char. } \\
\text { el. } \\
\text { length }\end{array}$ & $\frac{W_{c}}{t}$ \\
\hline 0 & S8R/STRI65 & 124542 & 1.49 & 0.5580 \\
$0^{*}$ & S8R/STRI65 & 167748 & 1.4 & 0.5685 \\
0 & S8R/STRI65 & 235152 & 1.26 & 0.5686 \\
45 & S8R/STRI65 & 126066 & 1.4 & - \\
90 & S8R/STRI65 & 105060 & 1.4 & - \\
\hline
\end{tabular}

Table 3.1: Overview of mesh refinement study

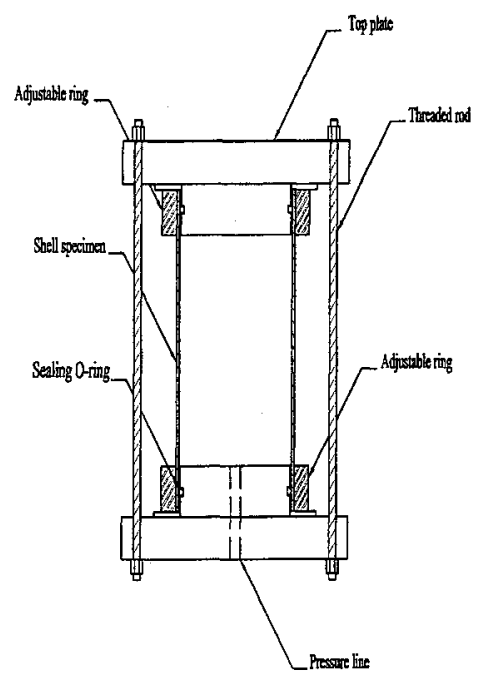

Figure 2.2: Schematic of shell setup.

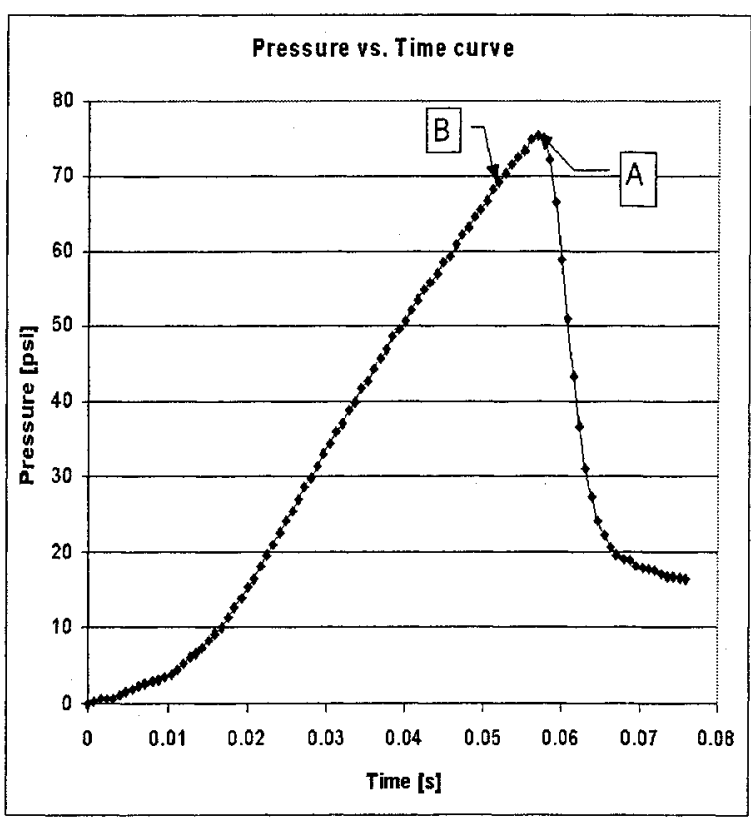

Figure 2.3: Typical pressure vs. time history plot for sudden pressurization during pressure build-up phase and global failure, for a shell with a crack at $\theta=60^{\circ}$. 

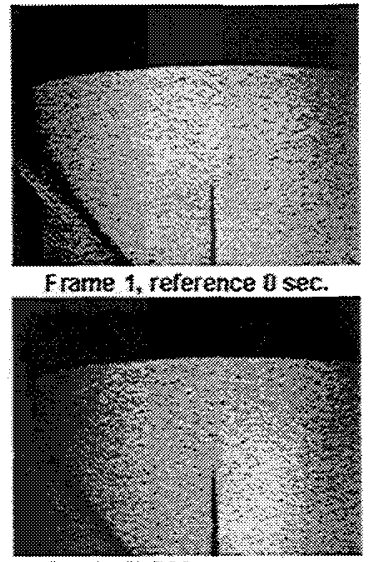

Frane 3,700 ysec

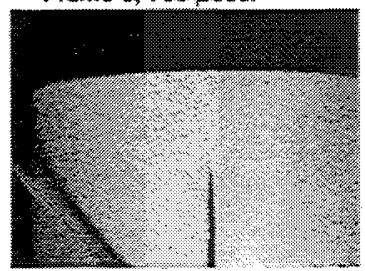

Frame 5,900 is sec

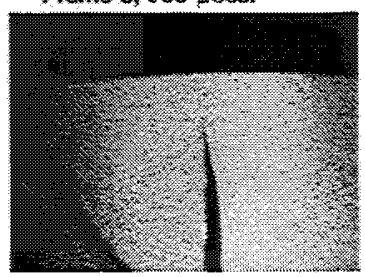

F rame $7,1100 \mu$ sec.

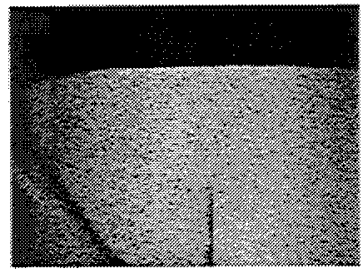

Frame 2, 400 usec.

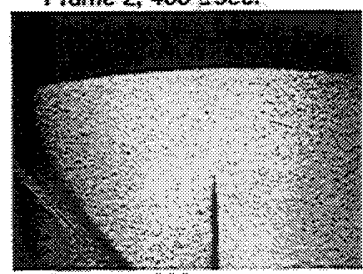

Frame 4,800 1sec.

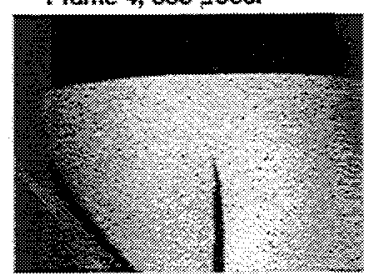

Frame 6,1000 isec

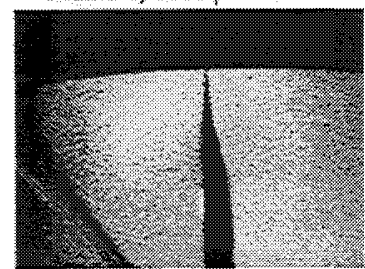

Frame 8, $1200 \mu$ sec.

Figure 2.4: Typical high speed camera images (100000 $\mathrm{Hz}$ ) of crack propagation of a 0.6 inch crack at 0 degree angle, $\lambda=3.40$.

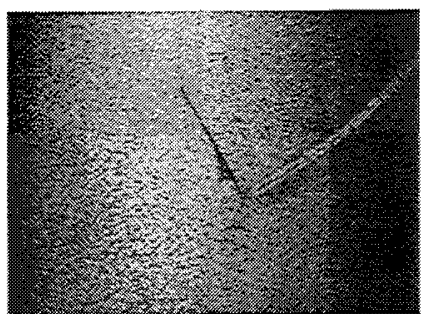

Frame 1: $2 a=0.886$ in $\{t=0\}$

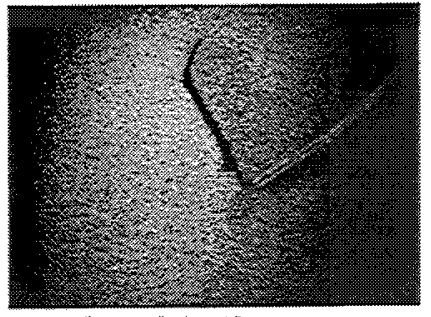

Frame $3:=40$ usec

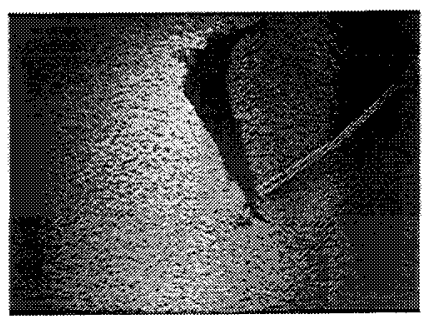

Frame 5: $t=60$ usec

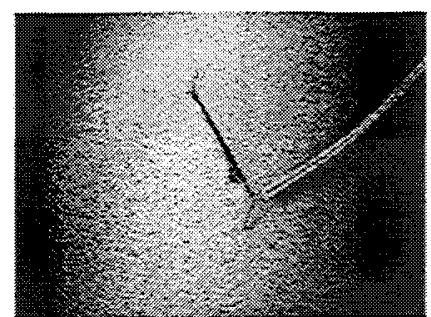

Frame $2:=30$ asec

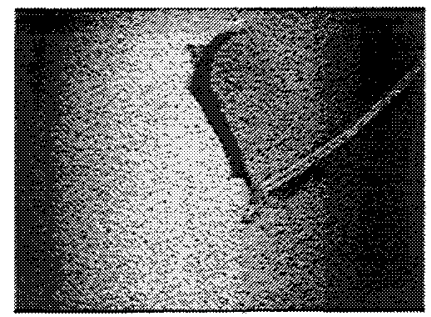

Frame 4: $:=50$ isec

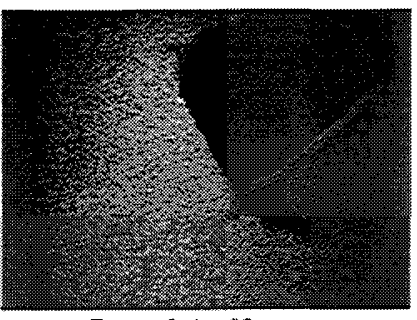

Frame $6: t=80$ isec
Figure 2.5: Typical high speed camera images of crack propagation of a 0.886 inch crack at 30 degree angle, $\lambda=5.0$.

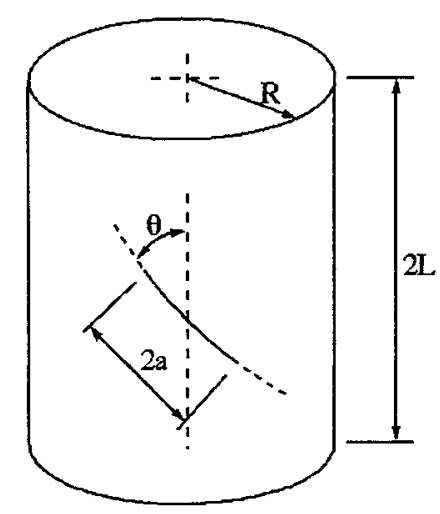

Figure 2.6: Schematic of shell parameter definitions. 


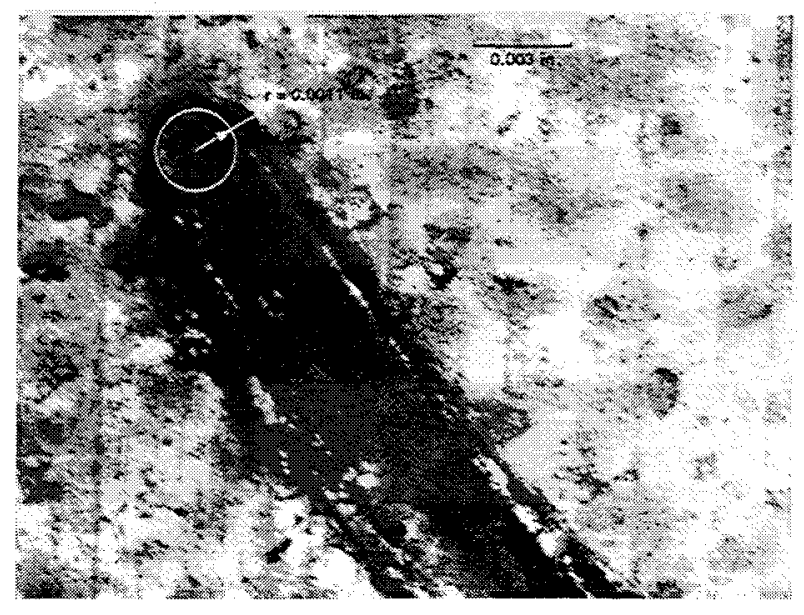

Figure 2.7: Microscopic image of crack tip.

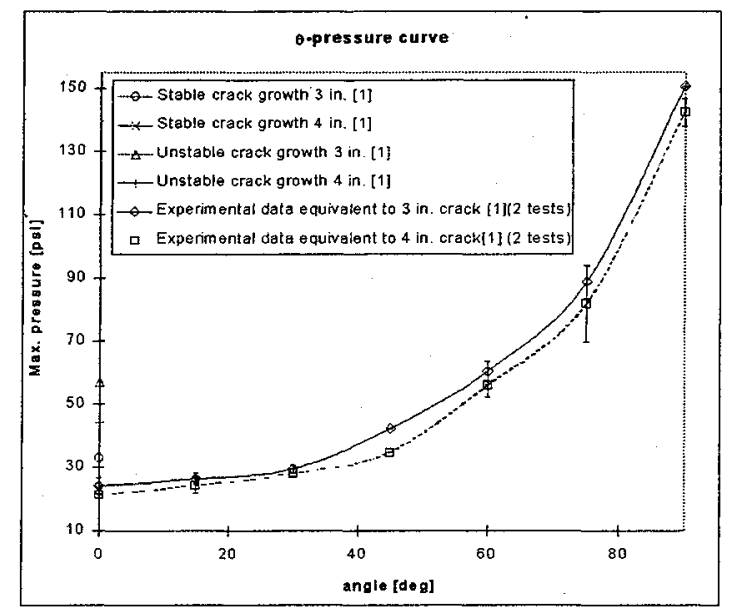

Figure 2.8: $\theta$-pressure curve.

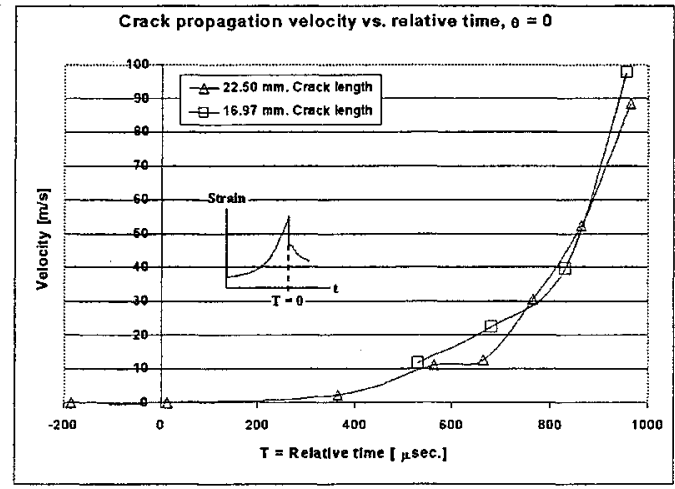

Figure 2.9: Crack propagation speed against crack growth increment.

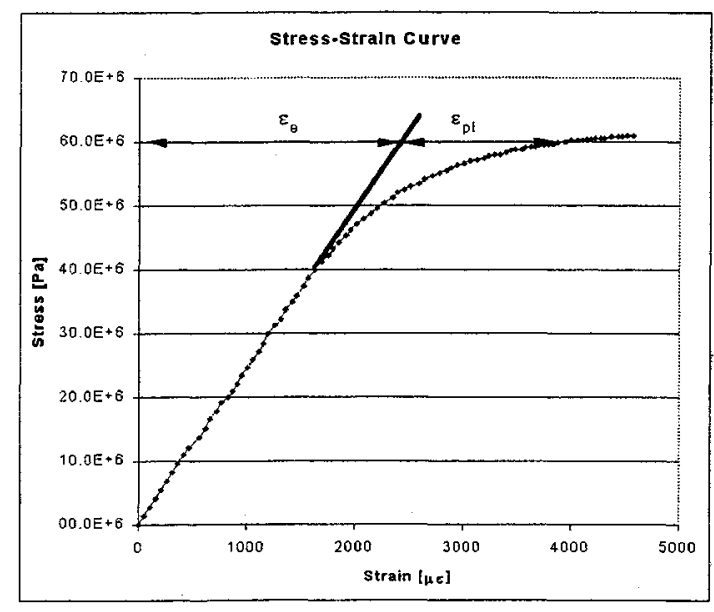

Figure 3.1: Stress-strain curve shell material.

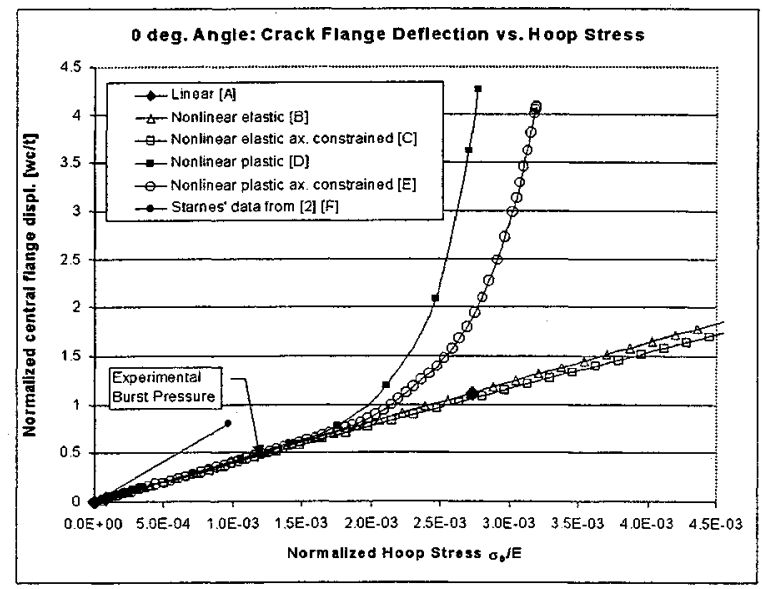

Figure 3.2: Finite element analysis results for 0 degree angle crack 


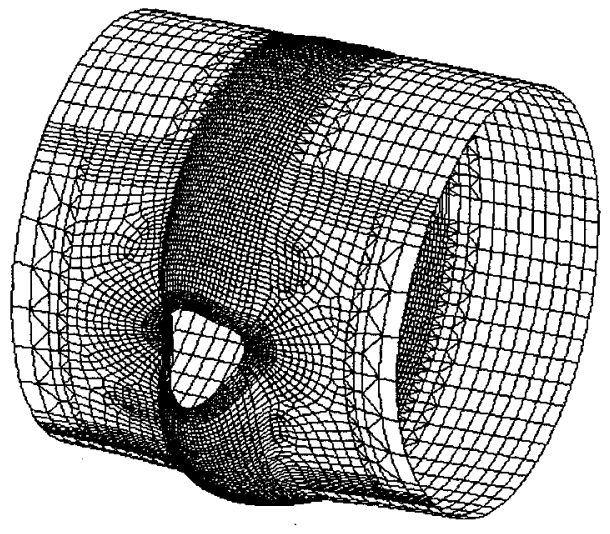

Figure 3.3: Deformed mesh for a shell with crack at 0 degree angle

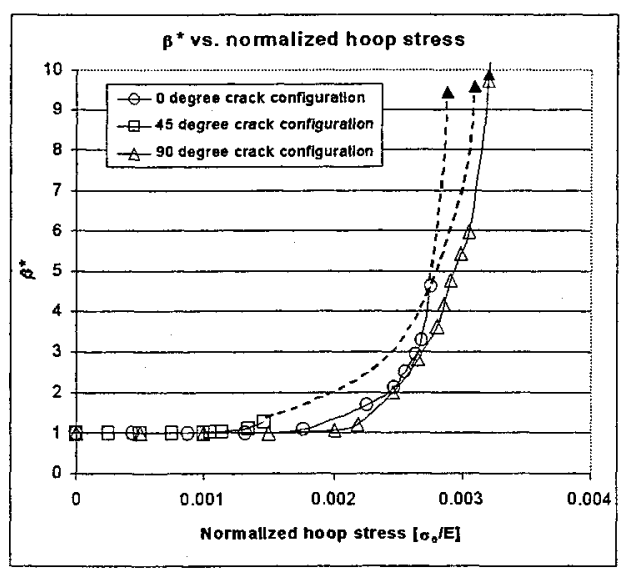

Figure 3.4: Degree of nonlinearity as function of the hoop stress

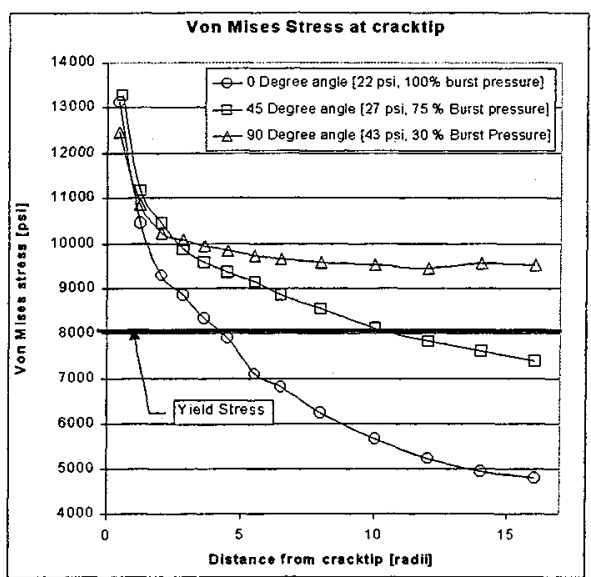

Figure 3.5: Von Mises stress vs. distance away from the crack tip

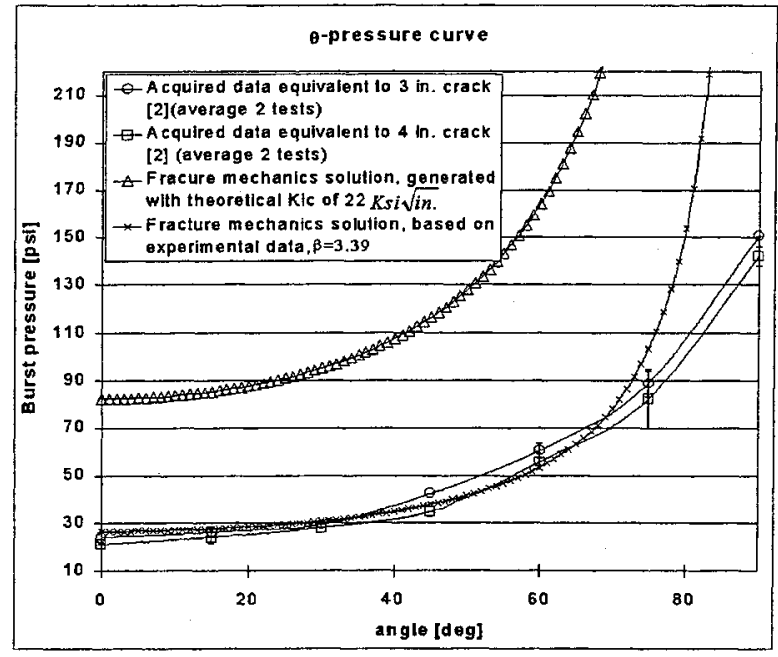

Figure 4.1: Comparison of results obtained from fracture mechanics and experimental data 


\section{References}

1. Hoogkamer, D., Waas, A.M. and Arbocz, J., "Damage Tolerance of Cylidrical Shells Containing Cracks.", Proceedings of the $41^{\text {st }}$ AIAA/ASME/ASC/ASCE Structures, Structural Dynamics and Materials Conference, April 2000, published by the AIAA.

2. Hoogkamer, D., Waas, A.M. and Arbocz, J., "Bulging Factors for Cracked Thin Shells: An Approximate Analytical Model.", Int.J.Nonlinear Mechanics, in review, 2001.

3. Veldman R.L., "Enhancing Commercial Aircraft Explosion Survivability via Active Venting.", Ph.D. Thesis Proposal, the Department of Mechanical and Aeronautical Engineering department at Western Michigan University, 1998, Kalamazoo, Michigan.

4. Chen, D., "Bulging of Fatigue Cracks in a Pressurized Aircraft Fuselage", Ph.D. Thesis, Delft University of Technology,Delft, The Netherlands, Report LR-647, October, 1990.

5. Jeong, D. Y., and Tong, P., "Nonlinear Bulging Factor Based on R-curve Data", Proceedings of the FAA/NASA International Symposium on Advanced Structural Integrity Methods for Airframe Durability and Damage Tolerance,September, 1994, pp. 327-338.

6. Bakuckas, J. G.,Jr., Nguyen, P. V., Bigelow, C. A., and Broek, D., "Bulging Factors for predicting Residual Strength of Fuselage Panels", Presented at the international Conference on Aeronautical Fatigue,Edinburgh, Scotland, June 18-20, 1997.

7. Starnes J.H. Jr., and Rose, C.A., "Nonlinear Response of Thin Cylindrical Shells with Longitudinal Cracks and Subjected to Internal Pressure and Axial Compression Loads", Proceedings of the $39^{\text {th }}$ AIAA/ASME/ASC/ASCE Structures, Structural Dynamics and Materials Conference, Vol. 3, AIAA, 1997, AIAA 97-1144.

8. Starnes J.H. Jr., Young R.D., and Rose, C.A., "Nonlinear Bulging Factors for Longitudinal and Circumferential Cracks in Cylindrical Shells Subjected to Combined Loads", Proceedings of the $41^{\text {th }}$ AIAA/ASME/ASC/ASCE Structures, Structural Dynamics and Materials Conference,Atlanta, Georgia, AIAA 2000-1514.

9. Rose, C.A., Young R.D., Starnes J.H. Jr., "Nonlinear Local Bending Response and Bulging Factors for Longitudinal Cracks in Pressurized Cylindrical Shells", NASA Langley Research Center Technical report under Grant NASA-CR-208454, Vol. 3, AIAA, 1997, pp. 2389-2402.

10. Rosakis, A.J., Samudrala, O., and Coker, D., "Cracks Faster than the Shear Wave Speed", Science, Vol. 284, AAAS, 21 May 1999, pp. 13371340.

11. Shahwan, Khaled W., and Waas, Anthony M., "Non-self Similar Decohesion along a Finite Interface of Unilaterally Constrained Delaminations", Proc. Royal Soc. Lond. A, Vol. 453, 1997, pp. 515-550.

12. ABAQUS/Standard Example Problem Manual version 5.7, Vol. 1, Hibbitt, Karlsson \& Sorensen Inc., 1997, Pawtucket, Road Island.

13. ABAQUS/Standard User's Manual version 5.7, Vol. 1, Hibbitt, Karlsson \& Sorensen Inc., 1997, Pawtucket, Road Island.

14. Dawicke D.S., Sutton M.A., Newman J.C. Jr., Bigelow C.A., "Measurement and Analysis of Critical CTOA for an Aluminum Alloy Sheet.", NASA Langley Research Center Technical Memorandum NASA-TM-109024, 1993, Hampton, Virginia.

15. Dowling N.E., "Mechanical Behavior of Materials: Engineering Methods for Deformation, Fracture and Fatigue.", Prentice Hall, 1999, New Jersey. 